\title{
AN INVERSE PDE-ODE MODEL FOR STUDYING BUILDING ENERGY DEMAND
}

\author{
Lianjun An \\ Young Tae Chae \\ Raya Horesh \\ Young Lee \\ Rui Zhang \\ IBM T.J. Watson Research Center \\ 1101 Kitchawan Road \\ Yorktown Heights, NY 10598, USA
}

\begin{abstract}
Development of an accurate heat transfer model of buildings is of high importance. Such a model can be used for analyzing energy efficiency of buildings, predicting energy consumption and providing decision support for energy efficient operation of buildings. In this paper, we propose a PDE-ODE hybrid model to describe heat transfer through building envelope as well as heat evolution inside building. A inversion procedure is presented to recover parameters of equations from sensor data and building characteristic so that the model represents a specific building with current physical condition. By matching the simulated temperature and thermal energy dynamic profile with EnergyPlus generated data and actual field data, we validate the model and demonstrate its capability to predict energy demand under various operation condition.
\end{abstract}

\section{INTRODUCTION}

The building sector, which includes commercial and residential buildings in the Unites States, accounts for $40 \%$ of the nation's total energy consumption (DOE 2006). It has also been responsible for $45 \%$ of the green-house gas (GHG) emissions. The prediction of energy usage in the building is useful for identifying opportunities for improving energy performance, saving energy consumption and for reducing greenhouse gases (GHG). However, it is difficult to develop a model that will accurately predict energy consumption in buildings because the energy consumption is influenced by building characteristics, HVAC systems, operations, occupants' behavior, weather condition and many other factors. Therefore, many approaches for building energy models that estimate building energy consumption in various conditions have been proposed and used from building design phase to operation and control and to retrofitting.

These approaches can be classified into two major types: forward and inverse modeling. Although the inverse modeling generally requires high level of mathematical techniques and expertise, it would be more feasible to predict energy consumption of existing building at a certain circumstance because the method needs a smaller set of input data comparing with the forward modeling and the key thermal parameters are deduced from actual building performance data (ASHRAE 2009). Dynamic inverse model as one of data-driven modeling for building energy performance is a method that allows estimation of thermal parameters of a building or space by using differential equations that describe the heat transfer phenomena (Andresen and Brandemuehl 1992).

A number of papers have been published for the dynamic inverse modeling, that aim at estimating key heat transfer parameters of building using sensor and meter data. Previous studies have applied a simplified thermal-network model (Wang and Xu 2006; Braun and Chaturvedi 2002; Fraisse et al. 2002) or thermal 
response model (Armstrong, Leeb, and Norford 2006), but many of them used simulated data to recover the thermal parameters or tested the model under a small laboratory setting (Park et al. 2011). One of the most common models is the simplified building internal mass model 2R2C (Schultz and Svendsen 1998), which from our experience, may not produce neither accurate nor robust prediction that are good enough for real buildings with real sensor data.

In this paper, we develop an integrated PDE-ODE model for describing heat transfer through a building envelope and thermal energy balance inside a building zone. Multiple terms in these equations are designed with multipliers used to adjust contribution of these terms. An inversion procedure is proposed to estimate these multipliers using sensor data from building management system (BMS) by minimizing the misfit between simulated and measured temperature. So that the tuned model represents a specific building under a current physical condition and these recovered effective coefficients reflect building current status due to aging and degrading. The procedure also addresses issues associated with avoiding local minimization and data over-fitting which often occurs in data mining processes and nonlinear programming. In fact, we are facing great challenge when dealing with data uncertainty coming from temperature dynamics simulation in finer time resolution inside a building. Weather (temperature, humidity, sky condition), wall surface and zone temperature, occupancy status and operation schedule are all sources of uncertainties in hourly resolution. Nevertheless, by comparing our simulation data with EnergyPlus generated data and actual field data, we can validate our model and demonstrate its capability to predict thermal energy demand under various conditions, for instance, for meeting a specified set-point during operation hours.

The paper is organized as follows. Firstly, we present a formulation of PDE-ODE model that describes heat transfer equations through the building envelope and interior zone. Secondly, we describes our calibration and simulation procedure with optimization technique. Thirdly, we validate the model by comparing the simulated result with EnergyPlus data, conduct calibration with field data from a commercial building and present simulation results with the recovered parameters under different conditions. Lastly, we provide conclusion and summary of the study.

\section{FORWARD PHYSICAL MODEL}

The enclosure, which includes wall, windows, floor and roof of a building (or a zone) provides insulation for the occupied space. Based on thermodynamics principles, heat can transfer through the enclosure (building envelope) in the form of conduction, convection and solar radiation.

Mathematically, wall temperature $T_{w s}$ for the side $s \in\{N, E, S, W, R\}$ satisfies the following PDE

$$
\rho_{w} C_{w p} d_{w s} \frac{\partial T_{w s}}{\partial t}=\frac{\partial}{\partial x}\left(\lambda_{k} K \frac{\partial T_{w s}}{\partial x}\right),(x, t) \in\left(0, d_{w s}\right) \times\left(t_{0}, t_{f}\right)
$$

with boundary conditions

$$
\begin{aligned}
& \lambda_{k} K \frac{\partial T_{w s}}{\partial x}(0, t)=-\lambda_{e h} h_{o s}\left(T_{a m b}(t)-T_{w s}(0, t)\right)-\lambda_{w s} Q_{s o l}(t)+\left(\lambda_{\text {eos }}-1\right) \sigma\left(T_{0}+T_{w s}(0, t)\right)^{4}, \\
& \lambda_{k} K \frac{\partial T_{w s}}{\partial x}\left(d_{w s}, t\right)=\lambda_{i h} h_{i s}\left(T_{z o n e}^{\text {sen }}(t)-T_{w s}\left(d_{w s}, t\right)\right)-\left(\lambda_{\text {eis }}-1\right) \sigma\left(T_{0}+T_{w s}\left(d_{w s}, t\right)\right)^{4} .
\end{aligned}
$$

The heat flux at wall surfaces consists of the convection driven by the difference between ambient air temperature and surface temperature, solar radiation on wall and heat radiated from wall. In Equation (1), $\rho_{w}$ is wall density, $C_{w p}$ is specific heat of wall, $h_{o s}, h_{i s}$ are convection coefficients which are functions of surrounding wind speed and temperature difference. $K$ is wall conductivity; $Q_{\text {sols }}$ solar radiation; $d_{w s}$ thickness of wall, subscript $s$ means that its value is different for each wall direction; $\sigma$ is the StefanBoltzmann constant. $T_{0}=273.15^{\circ} \mathrm{K}$ is the absolute temperature corresponds to $0^{\circ} \mathrm{C} . T_{a m b}, T_{z o n e}^{\text {sen }}$ are ambient and zone temperature respectively. Multipliers $\lambda_{k}, \lambda_{i h}, \lambda_{e h}$ are for conductivity and internal and external convection respectively. Note that no side of wall dependence is assumed here. Multiplier $\lambda_{w s}$ are for 
heat absorption coefficient, which is different for different sides, since each wall might be under different shading effect from nearby buildings or trees and with different color and smoothness. The multipliers $\lambda_{\text {eos }}, \lambda_{\text {eis }}$ are used to adjust external and internal wall heat radiation. Assuming we have a good estimation for the physical parameters, we expect all $\lambda$ 's value to be close to 1 . In such case, the entire factor for heat radiation could be negative. As a matter of fact, wall surface temperature reading from sensor is most likely to be inaccurate, and strongly depend on sensor location. We would like to treat this term as some kind of artificial term to handle a systematic and consistent error resulting from improper-emplacement of surface sensors.

Temperature $T_{z o n e}$ inside a zone satisfies the following ODE

$$
\begin{aligned}
& \lambda_{\text {ac }} \rho_{\text {air }} C_{a p} V_{\text {zone }} \frac{d T_{\text {zone }}(t)}{d t}=\lambda_{\text {inf }} \rho_{\text {air }} C_{\text {ap }} \dot{M}_{\text {inf }}\left(T_{\text {amb }}(t)-T_{\text {zone }}(t)\right) \\
& +\lambda_{\text {ih }} \sum_{s} h_{i s} A_{w s}\left(T_{w s}\left(d_{w s}, t\right)-T_{z o n e}(t)\right)+\sum_{s}\left(\lambda_{\text {eis }}-1\right) \sigma\left(T_{0}+T_{w s}\left(d_{w s}, t\right)\right)^{4} \\
& +\lambda_{g u} \sum_{s} U_{g s} A_{g s}\left(T_{\text {amb }}(t)-T_{\text {zone }}(t)\right)+\lambda_{\text {shgc }} \sum_{s} \lambda_{w s} Q_{\text {sol }} A_{g s}+\lambda_{\text {load }} Q_{\text {load }} \\
& +\rho_{\text {air }} C_{a p} \dot{M}_{\text {sys }}\left(T_{\text {sys }}(t)-T_{\text {zone }}(t)\right)
\end{aligned}
$$

where $\rho_{a i r}$ is density of air, $C_{w p}$ is specific heat of air, $V_{z o n e}$ is zone volume, $A_{w s}$ and $A_{g s}$ are areas of wall and window respectively, $U_{g s}$ is U-value of window, $\dot{M}_{i n f}$ is infiltration rate and $\dot{M}_{s y s}$ is AHU supply air flow rate. $Q_{\text {load }}$ is internal load, including contribution from lighting, electric equipment and occupants, $T_{s y s}$ is the system supply air temperature. Multipliers $\lambda_{a c}, \lambda_{g u}, \lambda_{i n f}, \lambda_{s h g c}, \lambda_{\text {load }}$ are for air heat capacity, for U-value of window, for air infiltration rate, for solar heat gain coefficient through window and for internal load respectively. The first term stands for air infiltration through building enclosure. The second and third terms in the right hand side represent convection and heat radiation contribution from different walls. The internal wall temperature $T_{w s}$ and the multipliers $\lambda_{i s}$ and $\lambda_{\text {eis }}$ were established during calibrating of the above PDE system. The fourth term stands for heat conduction through windows. The fifth term is for solar radiation contribution through window. The sixth term is for internal load, including heat contribution from lighting, equipment as well as occupants' body. The multiplier $\lambda_{\text {load }}$ can vary, depending on time of a day. A piecewise constant function is chosen with a constant value for every three hours in a day. The seventh term is for system supplied energy, which is used to maintain the comfort level of zone temperature. Note that heat contribution through window is modeled differently compared with the heat transfer through wall, and is modeled directly through the third and fifth terms in Equation (3), because window heat capacity is much smaller than wall heat capacity. The value of $\lambda_{a c}$ will reflect wall partition, furniture and equipment layout inside the zone. Both $\lambda_{g u}$ and $\lambda_{\text {inf }}$ are factors of terms containing $T_{s m b}(t)-T_{z o n e}(t)$, and would be correlated in current model.

We implement a numerical PDE solver for the parabolic equation using Crank-Nicolson scheme and an ODE solver using implicit Euler scheme. Both numerical algorithms for solving our PDE and ODE equations with given multipliers' value are unconditionally stable due to implicit nature on time-step evolution. The multipliers in these equations are used to adjust coefficients of various terms, since some of the specified values might not be accurate in current situation.

\section{INVERSION PROCEDURE}

In order to simulate thermal evolution inside a building, we need to establish multipliers' value. In this section, we describe our procedure to find out multipliers' value using real sensor data. Specifically, the multipliers will be a solution of certain minimization problem. The overall procedure is separated into two steps to overcome correlations among multipliers. 


\subsection{Minimization with PDE Constraint}

Inversion procedure for parameters estimation is posed as a minimization the objective function defined by

$$
\min _{\lambda_{i}} \sum_{k} \sum_{s}\left[\left(T_{w s}\left(0, t_{k} ; \lambda\right)-T_{w o s}^{\text {sen }}\left(t_{k}\right)\right)^{2}+\left(T_{w s}\left(d_{w s}, t_{k} ; \lambda\right)-T_{w i s}^{\text {sen }}\left(t_{k}\right)\right)^{2}\right]+\eta \sum_{i}\left(\lambda_{i}-1\right)^{2}
$$

by choosing proper multipliers

$$
\left\{\lambda_{i}\right\}=\left\{\lambda_{k}, \lambda_{i h}, \lambda_{e h}, \lambda_{w s}, \lambda_{\text {eos }}, \lambda_{\text {eis }} \| s \in N, S, E, W, R\right\}
$$

where $T_{w s}(x, t ; \lambda)$ is a solution of PDE defined in (1) with boundary conditions (2), $T_{\text {wos }}^{\text {sen }}(t)$ is sensor data of outside wall surface temperature and $T_{w i s}^{s e n}(t)$ is sensor data for inside wall surface temperature. The first term represents the sum of squared differences between simulated outside wall surface temperature and measured outside wall surface temperature - a misfit measure for outside wall surface temperature. Similarly, the second term represent the same misfit measure for inside wall surface temperature. Our goal is to find a set of multipliers so that the misfit defined by first two terms is minimized. In order to avoid over-fit to the noisy available data, a regularization term, the third term in the objective function (4), is included. Since those multipliers are multiplying factors of well-defined nominal physical value, they are expected to be close to one.

There are two major challenges in this optimization problem. First, our objective may not be convex function and a local minimum may be achieved. To address this, we randomize the initial guesses for these multipliers and perform the optimization multiple times. We choose the solution that obtained the minimal objective function value. Second, the solution may result from over-tuning the model from sensor data for the chosen period, since real sensor data is likely to contain errors and operation condition might be different from our expected one. The solution corresponding to the least misfit for specific period is not necessarily good solution for other periods and the misfit could become larger when applying the recovered multipliers on other periods. The regularization term helps us to address this issue through choosing a proper regularization coefficient. We first separate the collected data into two parts: training and validation sets. For each chosen regularization coefficient, a solution corresponding to the least objective value on the training set is found. Then the misfit value is calculated on the validation set with the solutions from different regularization coefficients. Finally, the solution corresponding to the least misfit on the validation set with certain regularization coefficient is chosen. Since it is impossible to test all different regularization coefficients over a large range, we pick the coefficient $\eta$ from a discrete set $\{0.1,0.01,0.001,0.0001,0.00001\}$ that covers different order of values.

Note that our objective function includes misfits from all walls, but subject to the same multipliers for conductivity and convection. In this way, we also reduce chance to over-fit certain biased sensor data. Even doing so, we might not fully resolve the over-tuning issue and will propose better statistical sampling in the conclusion section.

\subsection{Minimization with ODE Constraint}

The additional multipliers

$$
\left\{\lambda_{i}^{\prime}\right\}=\left\{\lambda_{a c}, \lambda_{\text {gu }}, \lambda_{\text {inf }}, \lambda_{\text {shgc }}, \lambda_{\text {load }}\right\}
$$

are chosen to minimize the following objective function, i.e.

$$
\min _{\lambda_{i}^{\prime}} \sum_{k}\left(T_{\text {zone }}\left(t_{k} ; \lambda^{\prime}\right)-T_{\text {zone }}^{\text {sen }}\left(t_{k}\right)\right)^{2}+\eta^{\prime} \sum_{i}\left(\lambda_{i}^{\prime}-1\right)^{2}
$$

where $T_{z o n e}\left(t ; \lambda^{\prime}\right)$ is the ODE solution of Equation (3) and $T_{z o n e}^{\text {sen }}(t)$ is sensor data of zone temperature. The first term in the objective (6) represents a misfit of zone temperature between simulated and measured and 
the second term is for regularization term. We use a similar procedure described in the last subsection to find these additional multipliers defined in (5). Note that, the multiplier $\lambda_{a c}$ could be greater than one, since there are partitioning walls and furniture inside a zone which are not being modeled, and the multiplier $\lambda_{\text {load }}$ is a function of time in a day.

\subsection{Stepwise Calibration}

The whole calibration procedure is shown in Figure 1. First, the minimization problem with PDE constraint is solved and a set of multipliers related to building envelope are recovered. Second, the minimization problem with ODE constraint is solved and additional multipliers are recovered to estimate internal load impact. The second minimization will use some information coming from the first minimization problem, like thermal energy consumption related to building envelope.

The left part of Figure 1 shows calibration procedure through minimization with PDE constraint. There are two loops in the procedure. The inner loop is used to get a solution with the least objective value on the train data for multiple random initial guesses with a given regularization coefficient in order to achieve a global minimum. The outer loop is used to get a solution with the least misfit on the validation data with different regularization coefficients in order to avoid an over-fitting situation. The right part of Figure 1 shows calibration procedure through minimization of the objective with ODE constraint.

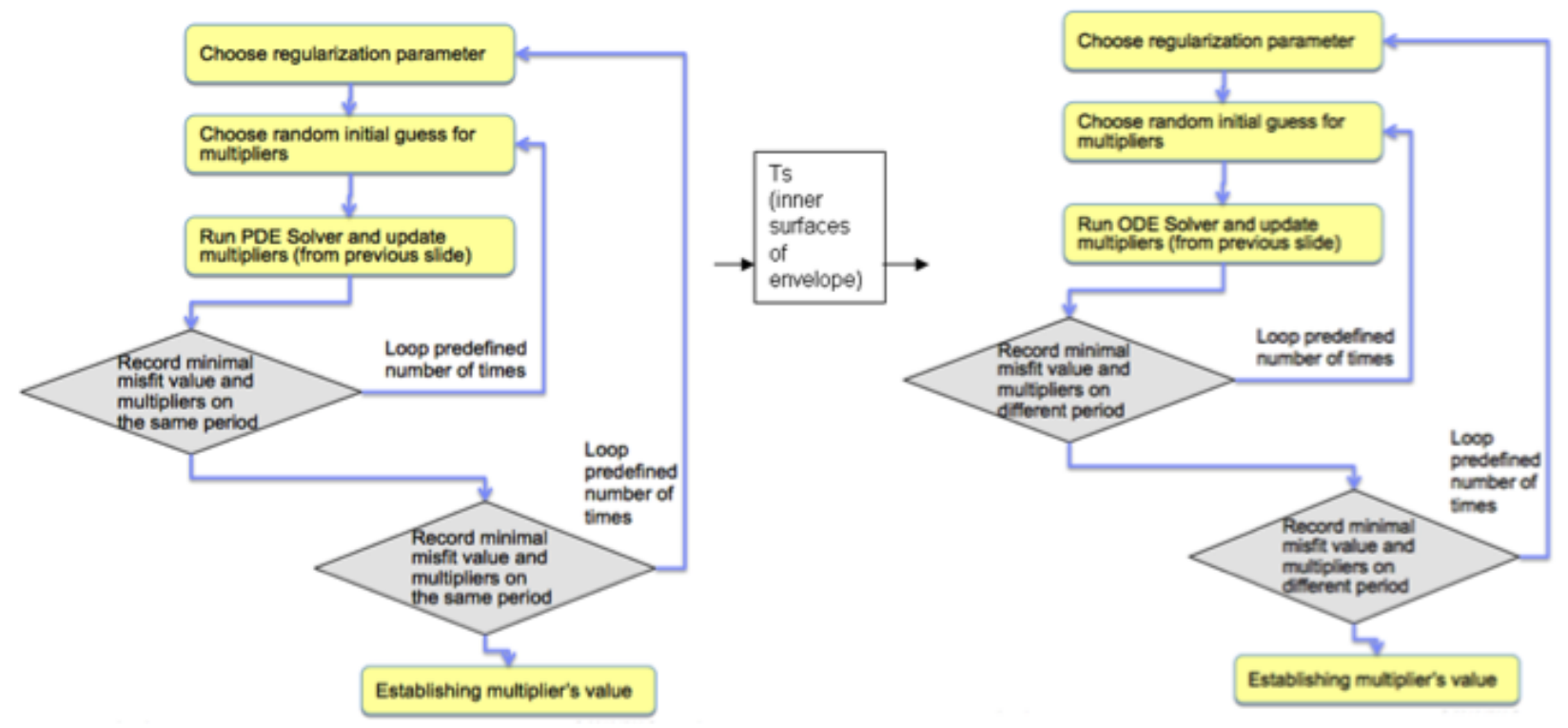

Figure 1: Stepwise calibration procedure.

When solving the minimization problem with PDE constraint, we not only estimate multipliers related to building envelope, but also find out heat-contribution to the internal walls to the zone. Heat gain or loss through building envelope will be substituted into the ODE model. With measured system contribution from AHU data, we can actually estimate internal load change in a day. In the other words, the load multiplier is found as a function of time in a day. In fact, our proposed stepwise calibration reduces impact from correlation among multipliers. It helps us to discover energy consumption distribution between building envelope and internal load more accurately. 


\section{SIMULATION METHOD}

After recovering multipliers through calibration using sensor data, we can use the integrated PDE-ODE model to simulate zone temperature dynamics and conduct what-if analysis, in addition different control strategies can be evaluated to meet comfortable level requirement.

There are two approaches for solving the system. The first approach is to solve PDE and ODE together and to obtain wall surface temperature and zone temperature under a given ambient temperature from weather forecast. The second approach is to solve PDE and ODE iteratively. First, with an initial guess of zone temperature, we solve the PDE and obtain internal wall surface temperature. Second, we solve the ODE with the internal wall surface temperature found from PDE and to obtain a new zone temperature. Then we solve the PDE again with the new zone temperature coming from solution of ODE. That procedure will be iterated several times until convergence.

When solving the ODE, we need to know AHU supply air flow rate and supply temperature $\dot{M}_{s y s}, T_{s y s}$ for these simulations. The purpose of air handling units is to adjust temperature and humidity inside a zone and maintain a comfortable climate for occupants. For a constant air volume (CAV) system, we only need to determine supply air temperature, In order to achieve this, we define the following objective function as

$$
\begin{aligned}
\operatorname{Func}\left(T_{\text {sys }}\right) & =C_{c p} \int_{t_{1}}^{t_{2}}(1-p(t)) \dot{M}_{\text {sys }}\left(T_{\text {sys }}(t)-T_{z o n e}\left(t ; T_{\text {sys }}\right)\right)^{ \pm} d t \\
& +C_{c p} \int_{t_{1}}^{t_{2}} p(t) \dot{M}_{\text {sys }}\left(T_{\text {sys }}(t)-T_{a m b}(t)\right)^{ \pm} d t \\
& +\mu \int_{t_{1}}^{t_{2}} \operatorname{sign}\left(\dot{M}_{\text {sys }}\right)\left|T_{\text {zone }}\left(t ; T_{\text {sys }}\right)-T_{s p}(t)\right|^{2} d t \\
& +\mu \int_{t_{1}}^{t_{2}}\left(1-\operatorname{sign}\left(\dot{M}_{\text {sys }}\right)\left|T_{\text {sys }}\right|^{2} d t .\right.
\end{aligned}
$$

Here $T_{z o n e}\left(t ; T_{s y s}\right)$ stands for zone temperature resulting from the ODE corresponding to a given $T_{s y s} . T_{s p}$ is a user specified temperature set point which may vary over time. $p$ is fresh air fraction when both ventilation and circulation are in operation and can be specified based on ventilation strategy. $\mu$ is the weight for meeting set point requirement. The first two terms represent thermal energy requirement and it can be either cooling or heating energy depending on either positive or negative part of the difference in the integrand. The first term represents air circulating, which is driven by the difference between return air temperature and supply air temperature $T_{\text {sys }}$; the second term represents air ventilation, which is driven by the difference between ambient air temperature and supply air temperature $T_{\text {sys. }}$. The third term in the objective measures the difference between zone temperatures and set point temperature. $\operatorname{sign}\left(\dot{M}_{s y s}\right)$ is a indicator function, whose value is 1 if $\dot{M}_{\text {sys }}>0$ and 0 otherwise. With this factor in the third term, it implies that set point temperature is targeted only during system being on. The fourth term is used to force $T_{\text {sys }}=0$ during system being off. By choosing an appropriate $\mu$ value, we balance between energy saving and comforting level of the zone. The objective function can be modified to incorporate other considerations, like humidity requirement, pre-cooling and pre-heating, and entropy control.

\section{PERFORMANCE EVALUATION}

We apply our forward model on a building model generated from EnergyPlus and validate our model by comparing the simulated temperature profile with the one from EnergyPlus. The proposed inversion procedure is applied on a medium size commercial building and the multipliers of equations corresponding to that building are estimated. The prediction is made based on our simulation method proposed in the previous section with given weather condition and specified set point. 


\subsection{Forward Model Validation with EnergyPlus Data}

In the forward model presented in Section 2, we include multipliers for different physical parameters. When parameters are chosen accurately, the solution from solving these equations with all multipliers being one should be consistent with the measured data. This is an important validation step through which the model accurately represents the physical process.

We treat the EnergyPlus model as an accurate simulation tool and create a building model and simulate its energy consumption with a given weather condition. The same weather condition and building characteristics are used in our PDE-ODE coupled model. The result coming from solving these equations with all multipliers being one is compared with the EnergyPlus results. Figure 2 shows the surface temperature of building's walls. The left one presents exterior wall temperature from both our model and EnergyPlus. The right one presents interior wall temperature also from both models. Correlation of wall temperature from both models reaches $99 \%$ and the root-mean-square error (RMSE) for exterior wall and interior walls is equal to 1.677 and 1.56 respectively.
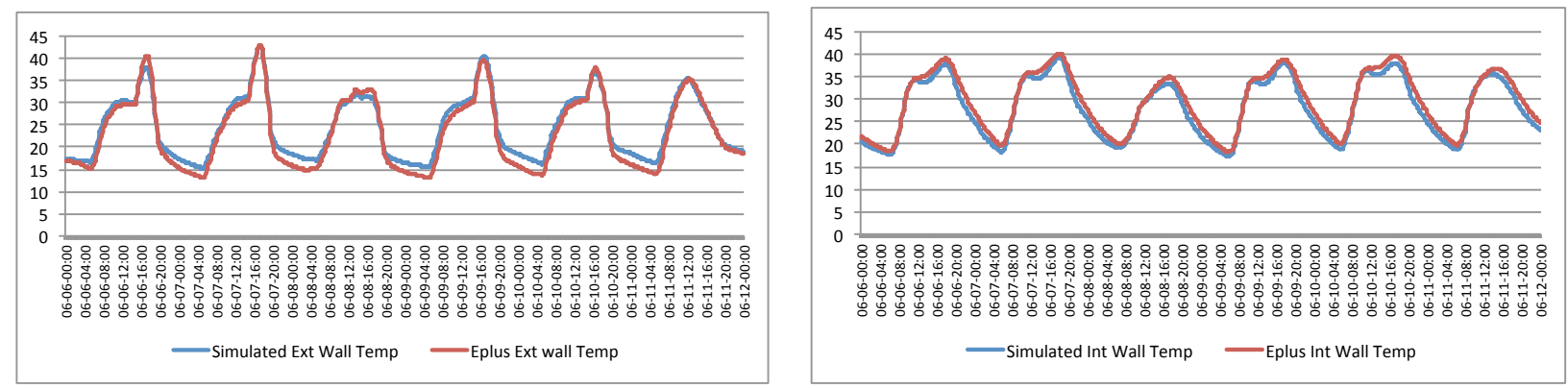

Figure 2: Comparison of wall temperature from our model and EnergyPlus.

During validating with EnergyPlus data, we create multiple EnergyPlus models, starting with the simplest one without window, infiltration, internal load and AHU system, and then adding influence factors back to the model one by one. The zone temperature obtained from solving the ODE specified in (3) also matches the zone temperature from EnergyPlus well with RMSE equal to 1.25.

\subsection{Calibration with Field Data}

When working with field data, the specified physical parameters are not accurate and the calibration procedure must be applied as described in Section 3. A medium-sized commercial office building was chosen for our study. This building was fully instrumented with wall surface temperature sensors, zone temperature sensor, in addition to supply air flow rate and temperature from the HVAC system. The recovered multipliers and the fitting accuracy are presented.

This building is located in Korea with 7800 squared meters and five stories. Both constant air volume (CAV) and electric heat pump (EHP) system were installed on each floor to meet the indoor thermal requirement of its space. Some physical and operational characteristics of the building are listed in Table 1. In addition to collecting local weather forecast data in 3 hours resolution from an on-line local weather station in Korea, overall 120 sensor or measurement data of both CAV and EHP systems, wall surfaces' temperature were collected and stored in a rational database. Note that, solar radiation data is not available in that weather station. Instead, a typical meteorological year data (TMY) for that location was adjusted based on the forecasted sky condition. Also, the assumption that the internal sensible load is the same during working days could be major source for inaccurate estimation. Wall surface temperature sensors are only deployed at the third floor, but their data are also used for other floors of the building. The supply air temperature from EHP was not available but was estimated from its return water temperature instead. 
Table 1: Building characteristics of test building.

\begin{tabular}{|c|c|}
\hline Exterior wall & $\begin{array}{c}\text { Gavanium STL plate }(0.0012 \mathrm{~m}), \text { Air cavity }(0.1 \mathrm{~m}) \\
\text { Insulation panel }(0.1 \mathrm{~m}), \text { Gypsum board }(0.012 \mathrm{~m})\end{array}$ \\
\hline Interior wall & $\begin{array}{r}\text { Gavanium STL plate }(0.0012 \mathrm{~m}), \text { Gypsum board }(0.012 \mathrm{~m}) \\
\text { Insulation panel }(0.03 \mathrm{~m})\end{array}$ \\
\hline Fenestration & $\begin{array}{c}\text { Gavanium STL plate }(0.0012 \mathrm{~m}), \text { Double pane clear glass } \\
\text { U value }=2.670 \mathrm{~W} / \mathrm{m} 2 \mathrm{~K}\end{array}$ \\
\hline Infiltration & $0.22 \mathrm{ACH}$ \\
\hline Occupancy density & $0.097 \mathrm{person} / \mathrm{m} 2$ \\
\hline Lighting density & $10 \mathrm{~W} / \mathrm{m} 2$ \\
\hline Plug load density* & $10 \mathrm{~W} / \mathrm{m} 2$ \\
\hline Operation schedule* & 6 a.m. -10 p.m. \\
\hline
\end{tabular}

Due to various error factors in the collected data, operation and internal load condition and assumption that might not be consistent with current situation, it is necessary to re-calibrate the model periodically. Specifically, the multipliers for internal load need to be re-estimated constantly in order to reflect the current daily running condition; some multipliers, like for wall thermal resistance, might not change very frequently unless some major retrofit activities just happened. Following the inversion procedure presented in Section 3, we conduct calibration of our model with sensor data in 15 minutes resolution that covers a week period in July, 2012 and the recovered multipliers' value are shown in the Table 2. It implies that U-value for window is slightly higher, with multiplication of 1.0539 , conductivity of wall is almost doubled with multiplication of 1.9018, heat capacity of air in the zone is tripled (might be reasonable due to impact of wall partition and furniture layout inside the zone). It is also notable that solar radiation impact on different side of wall is different due to certain shading effect from nearby buildings. Eight multiples (one for each three hours) for internal load are also recovered after balancing with envelope related energy consumption and supplied thermal energy from HVAC system. Figure 3 shows both specified load and the adjusted load for a workday. It can be seen that the adjusted load is higher than the specified, and load during evening hours is lower than the load during daytime from the adjusted load.

Table 2: Recovered multipliers' value.

\begin{tabular}{|c|c|c|c|c|c|c|c|}
\hline \multirow{2}{*}{ Name } & \multirow{2}{*}{ mUwin } & \multirow{2}{*}{ mCond } & \multirow{2}{*}{ mCair } & \multicolumn{4}{|c|}{$\mathrm{mSol}$} \\
\hline & & & & $\mathrm{N}$ & $\mathrm{E}$ & $\mathrm{S}$ & $\mathrm{W}$ \\
\hline Value & 1.0539 & 1.9018 & 3.6 & 0.5279 & 0.1396 & 0.0311 & 0.0311 \\
\hline
\end{tabular}

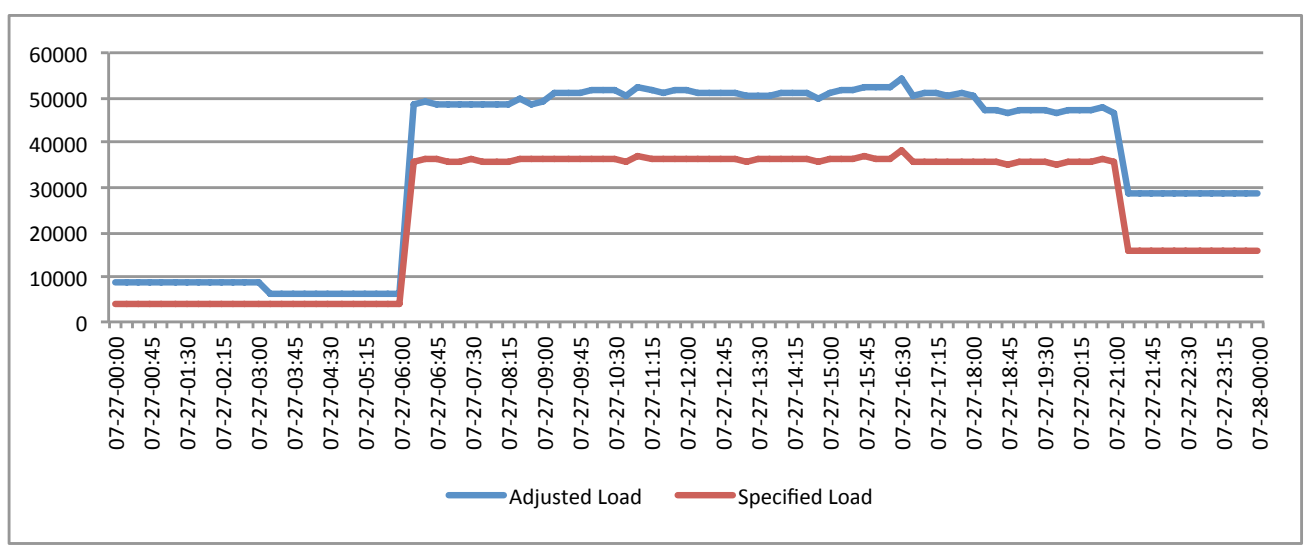

Figure 3: Comparison between specified and adjusted internal load. 
Figure 4 shows both simulated and measured temperature of the wall on the west side, the left is for outside wall and the right for insider wall. It can be seen that a reasonable match is achieved with 0.92 correlation and RMSE $=3.2$ for outside wall temperature and 0.98 correlation and RMSE $=1.68$ for inside. Also ambient temperature is included on the left and zone temperature is included on the right. It seems that the difference between inside wall temperature and zone temperature is too high. Recall the boundary condition for PDE, the internal heat radiation term with multiplier $\lambda_{\text {eis }}$ could used to correct certain bias. As long as that bias is also properly included in the zone balance equation, the thermal contribution through envelope can be correctly estimated. Figure 5 shows both simulated and measure zone temperature. Correlation of both temperature within seven days can reach 0.98 and its corresponding RMSE is equal to 0.1795 .
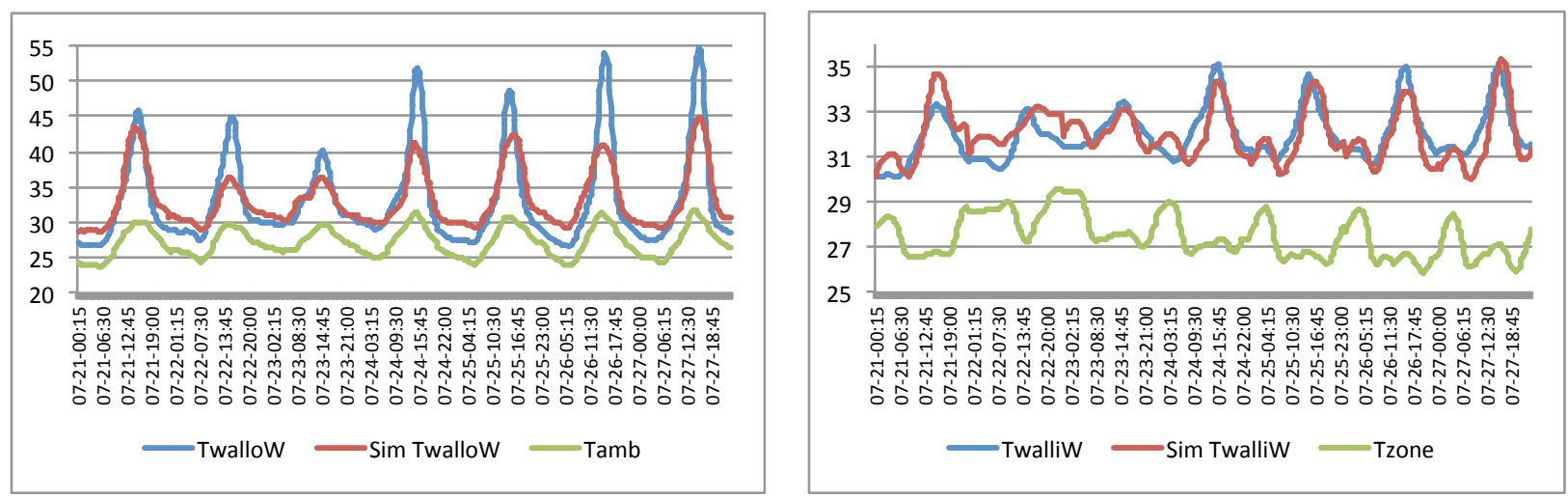

Figure 4: Comparison of wall temperature between simulated and measured.

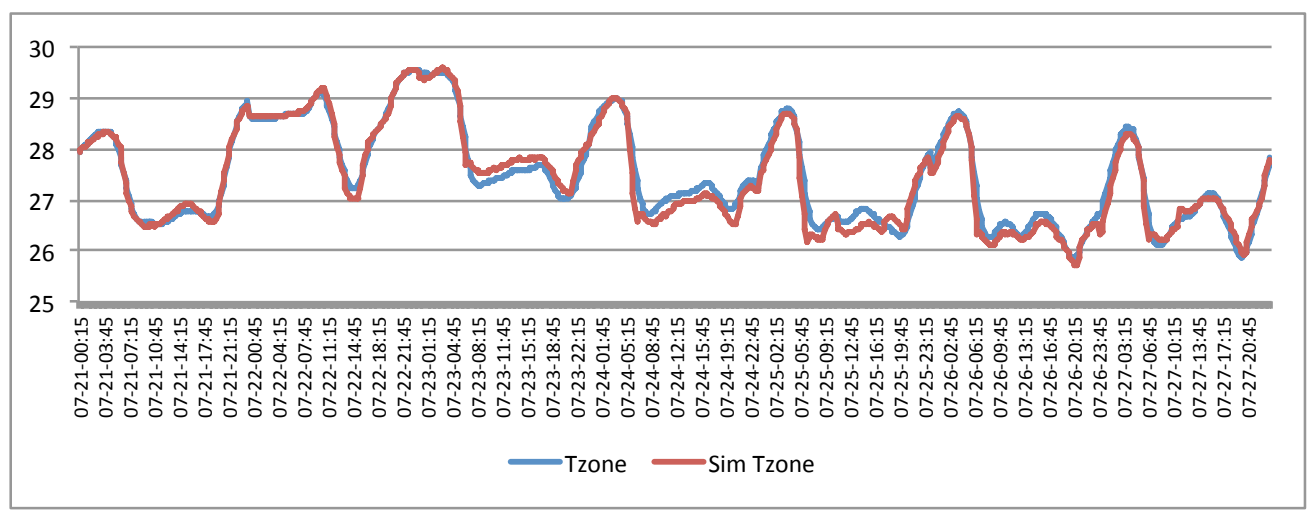

Figure 5: Comparison of zone temperature between simulated and measured.

\subsection{Forecast Under Various Conditions}

With the recovered parameters from the calibration, we can forecast demand thermal energy under given weather condition, zone temperature set-point requirement and operation schedule by using the method presented in Section 4. When the AHU system is of the CAV type, the supply air temperature (a set-point for chiller/or heater coil from plant) can be found as a solution of the optimization problem.

We use the recovered parameters in the last subsection, and forecast demand thermal energy on August $1^{\text {st }}$ in the following two ways: since the sensor data of outside air temperature and zone air temperature are available, the actual smoothed zone temperature (through HP filter) is chosen as set-point temperature and the actual operational hours is used for AHU operation. Figure 6 shows its simulation result. The 
simulation zone temperature follows the set-point well with 0.91 correlation and 0.44 RMSE. The accuracy drops near the operation starting point at 6 am in the morning. The predicted chiller supply air temperature and demand thermal energy also matches well with over 0.96 correlation. The demand thermal energy, as a ratio scale measure has $\mathrm{cv}(\mathrm{RMSE})=17.6 \%$. It is within the acceptable tolerance criteria $(\mathrm{MBE}+/-10 \%$, CV(RMSE) 30\%) of ASHRAE Guideline 14 (ASHRAE 2002).
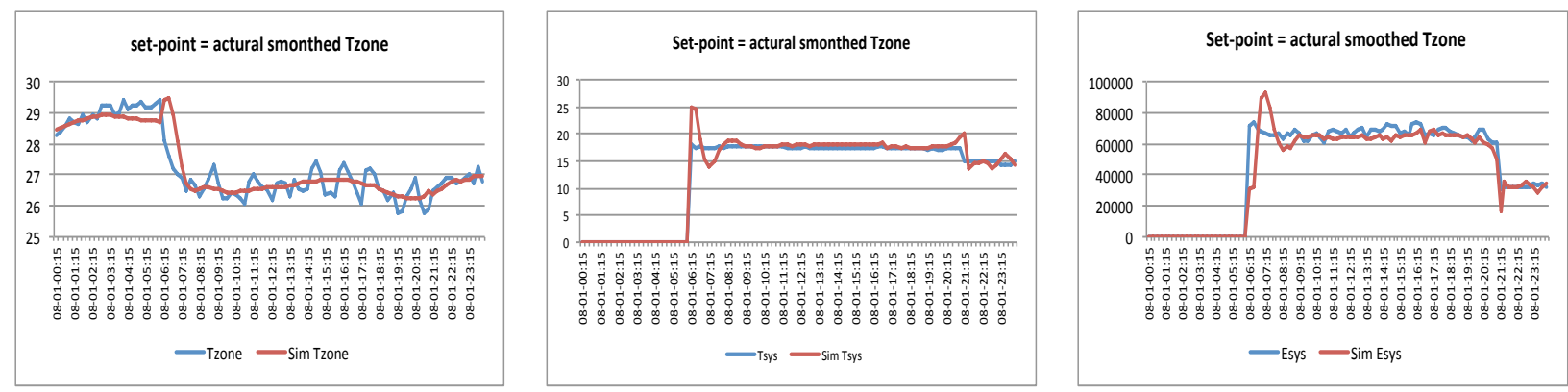

Figure 6: Simulation result with the actual smoothed zone temperature as set-point.

In the second test, we specify an operation schedule from 6 am to $9 \mathrm{pm}$, during which AHU is operating. The forecasted temperature from weather station is chosen as outside air temperature, and the set-point is specified to be $26^{\circ} \mathrm{C}$. Figure 7 shows its simulation result. It can be seen that the simulated zone temperature maintains the set-point during the specified operation hours. It is lower than actual zone temperature during the period, but is far off after operation hours since the AHU is not operating to maintain a comfortable zone temperature. The corresponding supply air temperature and thermal energy requirement are also shown in the figure. The required chiller set-point is a little lower compared to the actual $T_{s y s}$ due to the lower set-point compared to actual measured zone temperature during the operation hours.
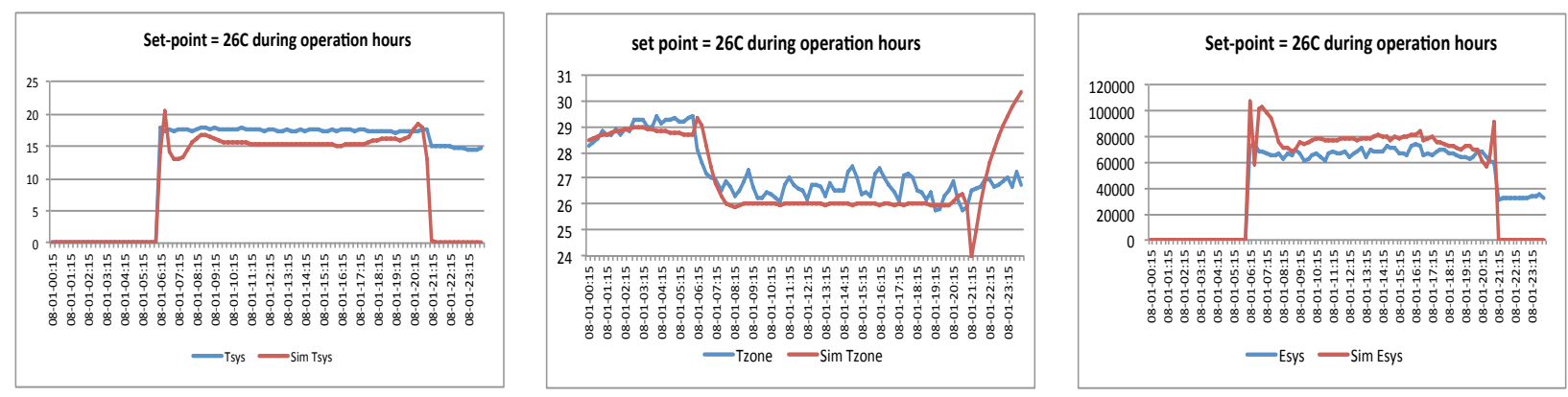

Figure 7: Simulation result with a given set-point and operation hours.

From the above simulation scenarios, we can conduct what-if analysis as well as forecast thermal energy demand. It is also possible to predict peak demand when a constant set-point is rigorously reached during operation hours (when less weight is put on saving energy in the objective given by Equation (7)).

\section{CONCLUSION}

We presented an integrated PDE-ODE model to describe heat transfer through building envelope and thermal balance inside zones. This model captures heat transfer phenomena in buildings more accurately than the reduced order models. Multipliers of parameters are introduced to the system and are estimated through a proposed calibration procedure. By defining an objective function with misfit term from all sides of walls and a regularization term, we improved the robustness of the procedure and avoided over-fitting to a certain set of sensor data. By separating calibration and parameters recovery procedure into two step processes, with first step being wall surface PDE calibration and second step being zone temperature ODE 
calibration, we reduced the probability for parameter correlation. Simulation procedure, that utilizes the PDE-ODE model with the recovered multipliers and simulated zone temperature control, is also formulated through optimization. This procedure generates energy demand profile under a given weather condition. We validated the model with EnergyPlus generated data, applied our inverse modeling approach to a commercial building in Korea. Thermal energy demand is predicted with calibrated parameters under various operating conditions. An initial study shows that the inverse modeling approach produces results with reasonable accuracy.

We plan to extend our work in the following two ways. Firstly, we would like to integrate our model with statistical tools and to get a distribution of the misfit value and obtain a response surface, based on the most likelihood calculation. When there are new sensor data becoming available, the respond surface would be updated using Bayesian updating procedure. In this way we can achieve the robustness of the inversion procedure. The simulation would not use a point value but use distribution associated with the parameters. Secondly, we would like to combine the analyses from different scales: thermal energy demand in daily level, and dynamical behavior in hourly level. Statistical forecast can generate daily level energy demand with reasonable accuracy. A PDE-ODE model simulates energy consumption profile in finer resolution than the statistical model. The objective function for the simulation consists of both aligning daily thermal energy consumption with the predicted one from statistical method and meeting hourly specified temperature set point.

\section{REFERENCES}

Andresen, I., and M. J. Brandemuehl. 1992. "Heat Storage in Building Thermal Mass: A Parametric Study". ASHRAE Transactions 98 (1): 910-918.

Armstrong, P. R., S. B. Leeb, and L. K. Norford. 2006. "Control with Building Mass - Part I: Thermal Response Model". ASHRAE Transactions 112 (1): 1-13.

ASHRAE 2002. Guideline 14-2002 - Measurement of Energy and Demand Savings. American Society of Heating, Refrigerating, and Air-Conditioning Engineers.

ASHRAE 2009. 2009 ASHRAE Handbook: Fundamentals. ASHRAE Handbook Fundamentals SystemsInternational Metric System. American Society of Heating, Refrigeration and Air-Conditioning Engineers.

Braun, J. E., and N. Chaturvedi. 2002. "An Inverse Gray-Box Model for Transient Building Load Prediction". International Journal of Heating, Ventilating, Air-Conditioning and Refrigerating Research 8:73-99.

DOE 2006. Buildings Energy Data Book. United States Department of Energy.

Fraisse, G., C. Viardot, O. Lafabrie, and G. Achard. 2002. "Development of a Simplified and Accurate Building Model Based on Electrical Analogy". Energy and Buildings 34 (10): 1017-1031.

Park, H., M. Ruellan, A. Bouvet, E. Monmasson, and R. Bennacer. 2011, oct.. "Thermal Parameter Identification of Simplified Building Model with Electric Appliance". In Electrical Power Quality and Utilisation (EPQU), 2011 11th International Conference on, 1-6.

Schultz, J. M., and S. Svendsen. 1998. "WinSim: A Simple Simulation Program for Evaluating the Influence of Windows on Heating Demand and Risk of Overheating". Solar Energy 63 (4): 251-258.

Wang, S., and X. Xu. 2006, April. "Simplified Building Model for Transient Thermal Performance Estimation using GA-based Parameter Identification”. International Journal of Thermal Sciences 45 (4): 419-432.

\section{AUTHOR BIOGRAPHIES}

LIANJUN AN is a researcher in the Mathematical Sciences Department of IBM T. J. Watson Research Center. He received Ph.D. degrees in applied mathematics from Duke University. He previously worked at McMaster and Stony Brook universities and conducted nonlinear analysis and parallel computation on partial differential equation models describing elastoplastic deformation and transport phenomena in porous media. He joined IBM in 1998 and has been working in the areas of demand forecasting and operation optimization 
and system dynamic modeling on supply chain, workforce management, finance, and healthcare systems. He currently works on physical inverse modeling for smarter buildings and evaluating building energy performance based on sensor data from building management systems. He has won the IBM Research Division Award and Outstanding Technical Achievement Award. His email address is alianjun@us.ibm.com.

YOUNG TAE CHAE is a postdoctoral researcher in the Mathematical Sciences Department of IBM T. J. Watson Research Center. He received B.S. and M.S. degrees in architectural engineering from Hanyang University and $\mathrm{Ph} . \mathrm{D}$. degrees in building science and environmental technology from the University of Illinois at Urbana - Champaign. He currently works on the development of a data-driven heat transfer model to evaluate building energy performance and to implement a decision-supporting system for building energy management. He has been one of the key developers of EnergyPlus, the official whole-building energy simulation program of the U.S. Department of Energy. He is a licensed professional engineer in HVAC and building mechanical systems in Korea. His research interests include computational modeling for HVAC components and building applications, building energy and occupants thermal comfort diagnostics, design and optimization of energy conservation measures for buildings, and performance enhancement of a hydronic radiant system. His email address is ychae@us.ibm.com.

RAYA HORESH is a research staff member in the Mathematical Sciences Department of IBM T. J. Watson Research Center. She received her B.S. degree in physics from Ben-Gurion University in Israel and her Ph.D. in applied mathematics from Emory University. She was an industrial postdoctoral fellow at the Institute for Mathematics and its Applications at the University of Minnesota. She joined IBM as a research staff member in 2011. Her research interests are in the fields of numerical analysis, numerical partial differential equations (PDEs), inverse problems, and optimization. In addition, she is particularly interested in the development of large-scale PDE-constrained optimization algorithms. Her email address is rhoresh@us.ibm.com.

YOUNG M. LEE is a research staff member in the Mathematical Sciences Department of IBM T. J. Watson Research Center. He received B.S., M.S., and Ph.D. degrees in chemical engineering from Columbia University. He currently leads an IBM research team on the development of smarter energy analytics for buildings and manufacturing plants. An IBM Master Inventor, he has won three IBM Research Division Awards and a BASF Technical Achievement Award for his mathematical modeling work. He is a frequent speaker at conferences, universities, and customer events. His research interest includes modeling, simulation and optimization of energy performance of buildings and manufacturing plants, supply chain management, manufacturing processes, services, workforce management, business processes, and emergency response operations. His email address is ymlee@us.ibm.com.

RUI ZHANG is a postdoctoral researcher in the Mathematical Sciences Department of IBM T. J. Watson Research Center. Before joining IBM, she got her Ph.D. from Carnegie Mellon University in the field of building performance and diagnostics. She received a dual B.Eng. in environmental engineering and computer science and an M.Eng. in environmental engineering from Tianjin University China. Her dissertation discussed the automated and advanced mesh generation for computational fluid dynamics (CFD) simulation in complex-free-form architectural designs and the coupling of building energy simulation with CFD. Her research interests include modeling, simulation and optimization of energy performance of buildings and manufacturing plants, numerical methods, data analytics, physical model development, and inverse modeling. Her email address is zhangrui@us.ibm.com. 